\title{
The future is bright
}

\section{Michel Matter}

Dr. med., Mitglied des FMH-Zentralvorstandes, Departementsverantwortlicher Dienstleistungen und Berufsentwicklung

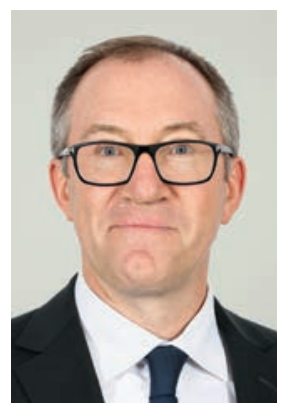

Die Feminisierung der Medizin ist augenfällig, und sie ist eines der Hauptthemen für künftige berufspolitische Entscheidungen. Für die FMH haben Grundlagen wie der ärztliche Nachwuchs, die Dauer der Weiterbildung, der Spagat zwischen Beruf und Privatleben und die Beschäftigungsquote Priorität. Diese Themen fassen die Zielsetzungen des Projekts «Coach my Career» zusammen, das diesen Herbst unter der Leitung von FMH und SIWF in Partnerschaft mit mfe, VSAO, VLSS und SWIMSA beginnt.

Der Aargauische Ärzteverband hat im Mai 2017 bei der Ärztekammer einen Antrag gestellt, um die beruflichen und familiären Bedürfnisse von Frauen besser zu berücksichtigen, die eine anstrengende Doppelrolle ausüben, und um es ihnen zu ermöglichen, Beruf und Privatleben dauerhaft in Einklang zu bringen. Die FMH gewährleistete über ihr Departement Dienstleistungen und Berufsentwicklung eine Koordinierung zwischen dem SIWF und den Initianten des Antrags. Anlässlich der Journée de réflexion des SIWF konnte dieses Thema im Januar 2018 umfassend behandelt und ausgearbeitet werden.

\section{Die Feminisierung der Medizin muss als} Chance für einen interprofessionelleren und weniger paternalistischen Ansatz verstanden werden.

Der Frauenanteil unter den FMH-Mitgliedern beträgt derzeit 39,5\%; in den Jahren 2016 und 2017 machten Frauen aber bereits 52,5\% der Neumitglieder aus. In einigen Kantonen hat sich die Kurve noch eindeutiger entwickelt, wie in Genf, wo in den Jahren 2016 und 2017 $60 \%$ der Neumitglieder Frauen waren (44\% der aktiven Mitglieder). Dieser Kanton wies schon früh eine Besonderheit auf: Zwischen 1900 und 1910 studierten hier mehr Frauen als Männer Medizin. Diese Tendenz endete mit dem Ersten Weltkrieg und der Schliessung der Grenzen. Erst im Jahr 2000 überschritt die Zahl der Studentinnen an der medizinischen Fakultät wieder diejenige der männlichen Studenten.
Die 1845 in Bözen (AG) geborene Marie Heim-Vögtlin, Pfarrerstochter und Mutter von drei Kindern, war die erste Ärztin in der Schweiz (Universität Zürich) und auch die erste Frau in Europa, die ihre eigene gynäkologische Praxis eröffnete. Die Post ehrte sie 2016 anlässlich ihres 100. Todestages mit einer Sondermarke. Erst in den 60er Jahren nahm die Anzahl der Frauen mit Medizindiplom wieder zu. Heutzutage haben sich die Verhältnisse umgekehrt: Der Frauenanteil liegt bei den Diplomierten nun bei über $60 \%$. Aber trotz der zunehmenden Feminisierung des ärztlichen Nachwuchses ist festzustellen, dass die verantwortungsträchtigen

\section{Bereits jetzt sind mehr als die Hälfte der Neumitglieder der FMH Frauen.}

Posten in den Diensten der Schweizer Spitäler, den Universitätsfakultäten oder den wichtigsten medizinischen Zentren in allen Disziplinen weiterhin in männlicher Hand sind. Im CHUV gibt es beispielsweise $60 \%$ Assistenzärztinnen, aber lediglich 27\% werden Kaderärztinnen und nur 12\% Professorinnen. Schwierigkeiten bereitet die Lebensphase zwischen 30 und 40 Jahren, wenn berufliche und private Lebensentscheidungen anstehen.

Die Arbeitsbedingungen müssen in allen Disziplinen aus Überzeugung und mit Dynamik an die aktuellen Bedürfnisse der Ärztinnen und Ärzte angepasst werden. Die Medizin hat nicht genug Flexibilität an den Tag gelegt, um Reformen durchzusetzen, die unter Berücksichtigung der beruflichen und privaten Bedürfnisse einen hochqualifizierten medizinischen Nachwuchs gewährleisten. Die Weiterbildung muss angepasst werden, um endlose Berufsausbildungen zu vermeiden, Bereitschaftssysteme müssen überdacht und die Teilzeitarbeit flexibler gestaltet werden. Das Schicksal unseres Berufsstands und der Patientenbetreuung liegt in den Händen der neuen Generation. Es gilt also, den Zusammenhalt zwischen den Generationen zu pflegen. Denn darin liegt unsere Stärke. 